This item was submitted to Loughborough's Research Repository by the author.

Items in Figshare are protected by copyright, with all rights reserved, unless otherwise indicated.

\title{
A new way of understanding the customer, for fibre manufacturers
}

PLEASE CITE THE PUBLISHED VERSION

PUBLISHER

(C) Emerald

LICENCE

CC BY-NC-ND 4.0

REPOSITORY RECORD

Lofthouse, V.A., T.A. Bhamra, and T. Burrow. 2019. "A New Way of Understanding the Customer, for Fibre Manufacturers". figshare. https://hdl.handle.net/2134/1006. 
This item was submitted to Loughborough's Institutional Repository by the author and is made available under the following Creative Commons Licence conditions.

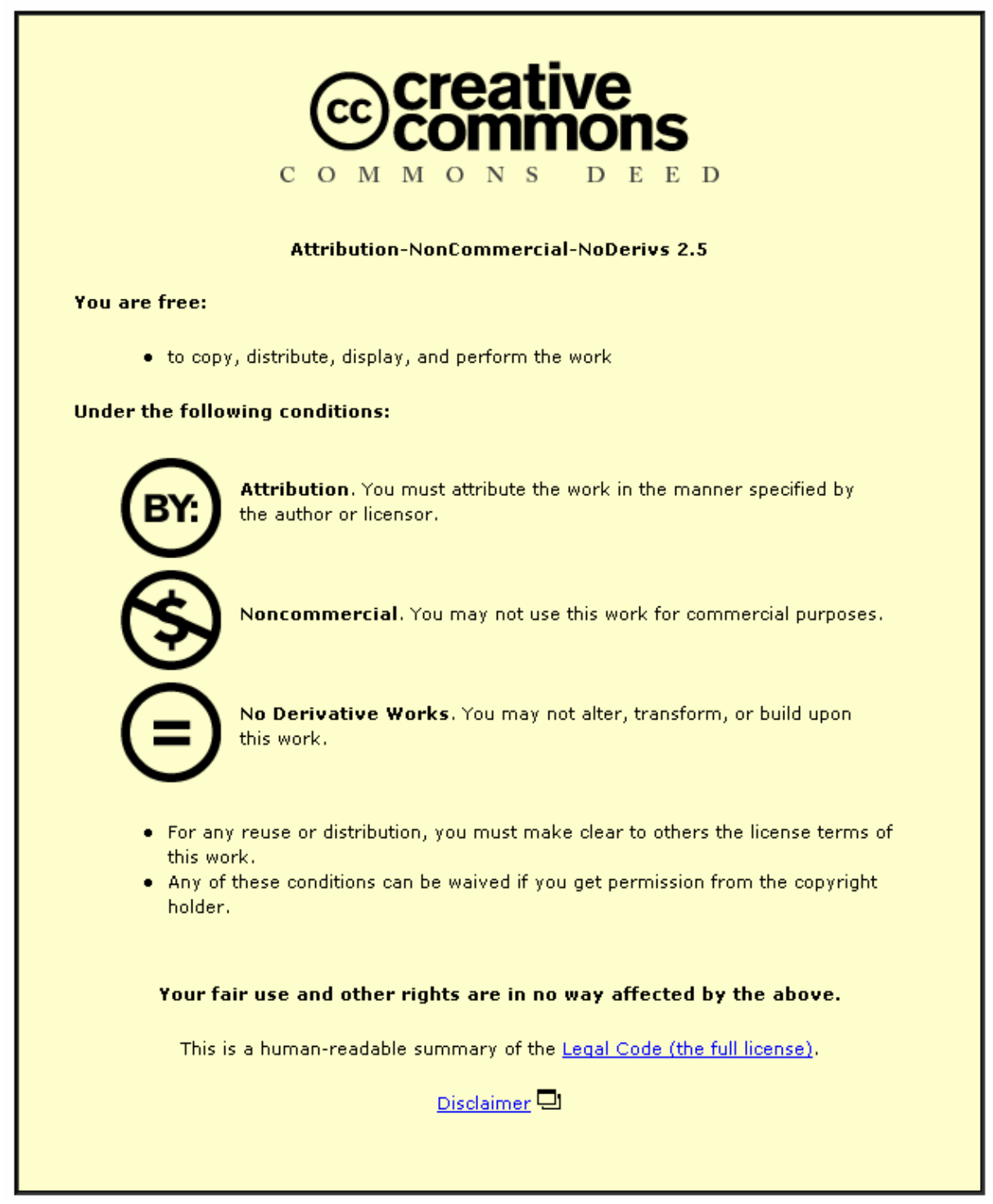

For the full text of this licence, please go to: http://creativecommons.org/licenses/by-nc-nd/2.5/ 


\title{
A new way of understanding the customer, for fibre manufacturers
}

\author{
Vicky Lofthouse*, Tracy Bhamra* \& Tom Burrow** \\ * Department of Design \& Technology, Loughborough University, Leicestershire, LE11 3TU \\ ** Tencel Ltd, Spondon, Derby, UK
}

Key words: Textiles, Tencel, Fibre manufacturer, Visual mapping, Empathic Design Techniques, Kano Word count of main text: 4786

\begin{abstract}
This paper describes a collaborative research project run as part of the UK based Textile \& Clothing Industry Forum, that aimed to investigate new ways of understanding the end customer. In deciding to 'speak' to the end customer, the fibre manufacturer Tencel Limited made a radical move to break away from the traditional structure of the apparel supply chain. This paper describes how a combination of a Kano questionnaire and empathic design techniques developed for the automotive industry were effectively used to gather new insights into how customers really perceived Tencel's fibre, and enable the product development team to 'walk in the shoes' of the people they design for. The paper concludes that applying these techniques to the textiles industry is a very effective way of obtaining rich and genuine opinions from the end customer and saw that the 'raw' video data generated proved to be a very successful way of communicating with senior management.
\end{abstract}

\section{Introduction}

This paper describes the novel approach taken in a collaborative research project that aimed to investigate new ways of understanding the customer, for Derby based fibre manufacturer, Tencel Limited. The project was carried out between September 2001 and October 2002 as part of the UK based Textile \& Clothing Industry Forum. This initiative funded, by the UK Department of Trade \& Industry (DTI) and companies from the textile \& clothing industry, aimed to demonstrate and facilitate the delivery of profitable supply chains in partnership with UK manufacturers and retailers, and to develop the processes for sustaining competitive advantage (Fashion Industry Forum, 2002). The remit of the Textile \& Clothing Industry Forum (TCIF) is to organise "live" projects, which are carried out within the workplace with participating retailers and suppliers. One of their specific aims is to take technologies and approaches which have a proven track record in other industries (such as automotive and electronic product manufacturing) and adapt them for use within the textiles and clothing industry (Bhamra et al., 1998).

The overall aim of the research described in this paper, was to help identify and establish a significant retail programme with a major UK store group for Tencel limited. In 1978, Courtaulds Fibres began research into solvent spun cellulosic fibre technology in response to predicted market changes. The resulting fibre TENCEL ${ }^{\circledR}$, a registered trademark of Tencel Limited for Lyocell (Tencel Ltd., 2002), was launched in 1992 claiming to be the first new fibre to be introduced on the market for 30 years (Curtis, 1997). Tencel Limited is currently the world's leading supplier of Lyocell, with an $80 \%$ share of the market and an annual turnover of approximately 100 million Euros. Over the last 10 years a new fibre variant has been developed - Tencel A100, ideally suited for use in knitted applications. The new fibre has proven to be very successful in jersey fabrics but has only achieved success in limited geographical markets for knitwear, and developments in western markets particularly have been slow. Initial research by TCIF suggested that problems with the perception of the Tencel fibre existed at several levels within the supply chain. Within processing companies, it was perceived that there would be technical difficulties regarding processing the fibre into yarn and this was a valid reason for them not to 'push' A100 through the supply chain. At the retail level of the supply chain there was a perception that garments manufactured from Tencel would be more expensive to produce than products made from equivalent yarns (Lofthouse and Thomas, 2002). 
In order to move beyond the problems outlined above, Tencel Limited and the TCIF team set out to gain an understanding of the end customer's (i.e. the consumer) perception of A100, based on the belief that this would provide a convincing package of evidence to illustrate to buyers that customers wanted garments knitted in this fibre variant. It was felt that strong support from the end user would help to 'pull' the fibre through the supply chain. This paper describes the approach adopted in order to gain an understanding of the end customers' perception of Tencel's product and how this knowledge was used to create a package of evidence that could be used to demonstrate the positive features of their product.

\section{Moving beyond the traditional}

In deciding to 'speak' to the end customer, Tencel were making a radical move and breaking away from the traditional structure of the apparel supply chain. Figure 1 illustrates this change, showing the traditional approach to the textiles supply chain which relied on yarn being 'pushed' through via the fibre manufacturers. This is compared to the new approach adopted in this project which set out to empower Tencel to utilise their customer understanding to 'pull' the yarn along the supply chain. It was believed that this would allow them to gain confidence in their downstream customers and demonstrate that they have a product that satisfies an end-user need.

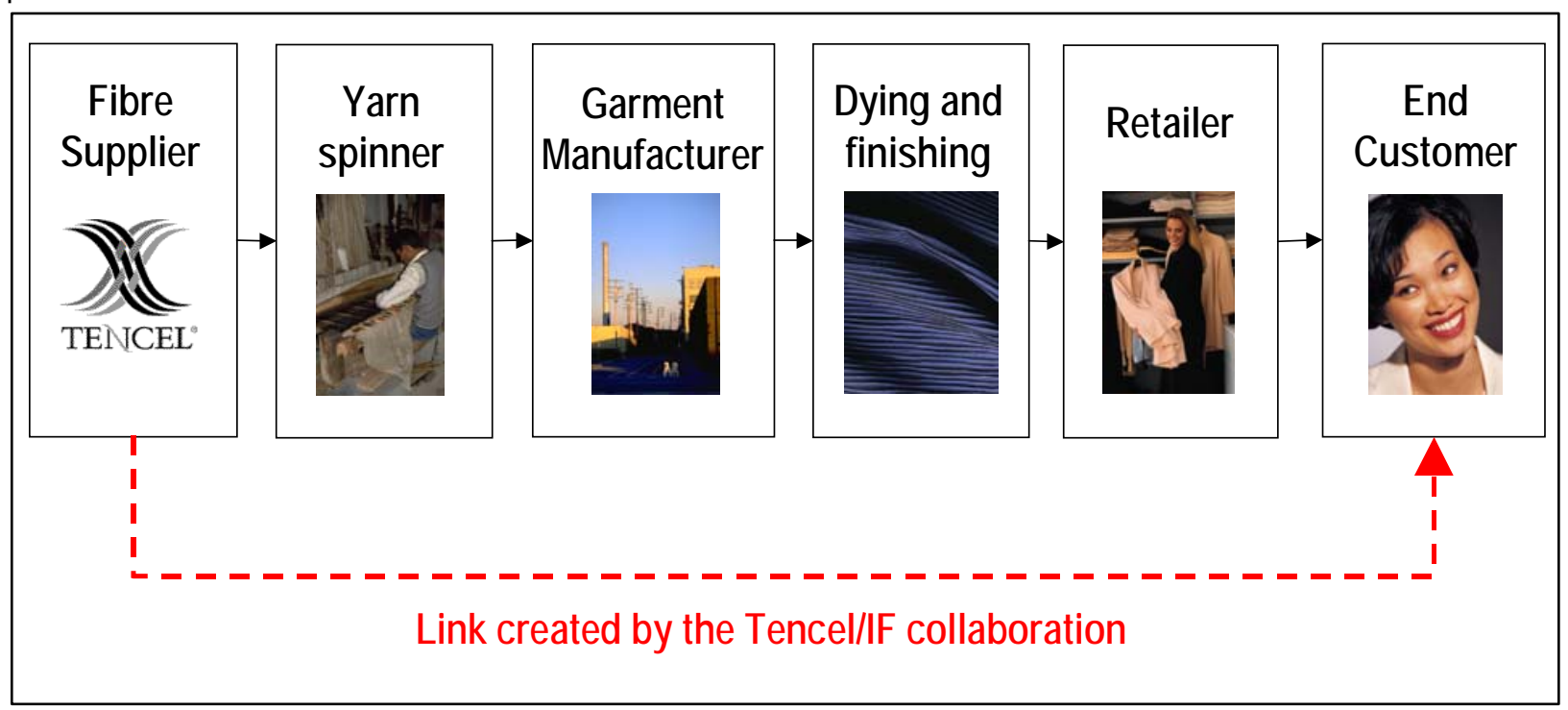

Figure 1 Supply chain diagram

Setting out to better understand the end customer was an unaccustomed approach for the technical team of a fibre manufacturer that traditionally focused on science and technology rather than the 'touchy feely' side of human emotions. In the traditional set-up a fibre supplier like Tencel would only speak to the manufacturer of the textile product, and these sorts of conversations would have a very technical/ scientific basis. To be able to effectively communicate with the end customer (non technical shoppers) the Tencel team would have to learn a whole new vocabulary and develop new ways of talking about knitwear features. In order to support this investigation, tools and techniques which were developed to investigate emotional responses to design features in the automotive industry, were adapted for use within the textiles industry (Burns et al., 1999, Burns and Evans, 2002, Burns et al., 2000, Burns and Evans, 2000). These tools are described in more detail in section 4. In addition to these data collection techniques, an approach developed by The Grove Consultants International (2003) was adapted to help with the management of this complex and intricate project. The Grove Consultants International is a process consulting firm that has pioneered the use of visual approaches to help facilitate Strategic Visioning in collaborative work environments. Their techniques aim to stimulate participation, encourage teams to think about the 'big picture' and enhance group learning and memory (The Grove Consultants International, 2001). 
This paper outlines the tools which were used throughout the Tencel/TCIF collaboration and illustrate how they benefited the project team and helped Tencel to generate their first insight into the perceptions that their end customers have of their product offering.

\section{Managing the project}

The Tencel/TCIF project was carried out by a multidisciplinary team, located at three different sites in the UK. It consisted of two members of the Industry Forum team, a project leader who managed the project and a research assistant with comprehensive experience of the textiles industry and of running focus group activities. These team members were based at Cranfield University, (now at Loughborough University). The multidisciplinary Tencel team consisted of the Fabric Development Manager, the Knit Specialist, the Retail Account Manager, the Dyeing and Finishing Development Manager and the Support Unit Manager. The majority of the Tencel team were based at the Spondon site in Derbyshire, with the exception of the Retail Account Manager who was based in their London offices.

One of the key aims of the Tencel/TCIF project was to ensure that at the end of the project the Tencel team had acquired the skills and knowledge to run similar projects in the future. In order to support this the whole team were involved throughout the project and were actively engaged in decision making and in developing activities such as those used in the focus groups. Although the TCIF team ultimately managed the project and also presented suggestions for activities and the structure of the focus groups, these inputs were always discussed and manipulated by the whole team to ensure that the best possible result was obtained. Consequently each member of the team had a degree of responsibility for the outcome of the project and a stake in its success.

In order to achieve a high degree of team working, manage the project effectively across the different geographical locations and make effective use of the different expertise available, a 'new' tool was introduced at the start of the project. A visual communication tool, adapted from a template entitled 'Your Company's Gameplan' designed by The Grove Consultants International (2003) was used to plan the project and record the progress of the team. Despite being originally developed for strategic visioning existing templates can be adapted or developed for a variety of business purposes (i.e. business planning, creative group work) and have been shown to be an effective way of capturing and sharing the thoughts of group members, and for keeping concepts alive long after the meeting. (Young et al., 2003). The adapted graphical template was known as the 'Journey Diagram', was eight feet by four feet in size and consisted of four main areas. A section on the left hand side of the diagram entitled 'Resources' recorded the team members names along with any external resources that could be drawn upon. The main section, which was illustrated by a large blue arrow that indicated the direction of travel, recorded the deliverables and tasks which needed to be completed. A large yellow target on the right hand side of the diagram reminded the team of the ultimate aim of the project and helped to reduce deviation from the goal. At the bottom of the diagram green hills provided the team with an area in which to record the challenges that faced them during the project. A photograph of Tencel's 'Journey Diagram' being used as a work in progress is shown in Figure 2.

The 'Journey Diagram' was used to record all of the activities that the team identified were necessary to progress the project towards the final target. Tasks were discussed at regular team meetings, recorded on individual Post-it Notes and stuck onto the plan. The flexibility of this approach meant that activities could be moved around and reordered until the team were happy. At each meeting, the Journey Diagram was fixed to the wall and used for reference and further planning. Activities which should have been completed were reported on and ticked off when they were finished with. Future activities were identified and assigned an owner and a completion date. Throughout the project, the template was used during meetings and then photographed and distributed to the team shortly after each meeting, along with comprehensive minutes which recorded all major decisions and the reasoning behind them. 


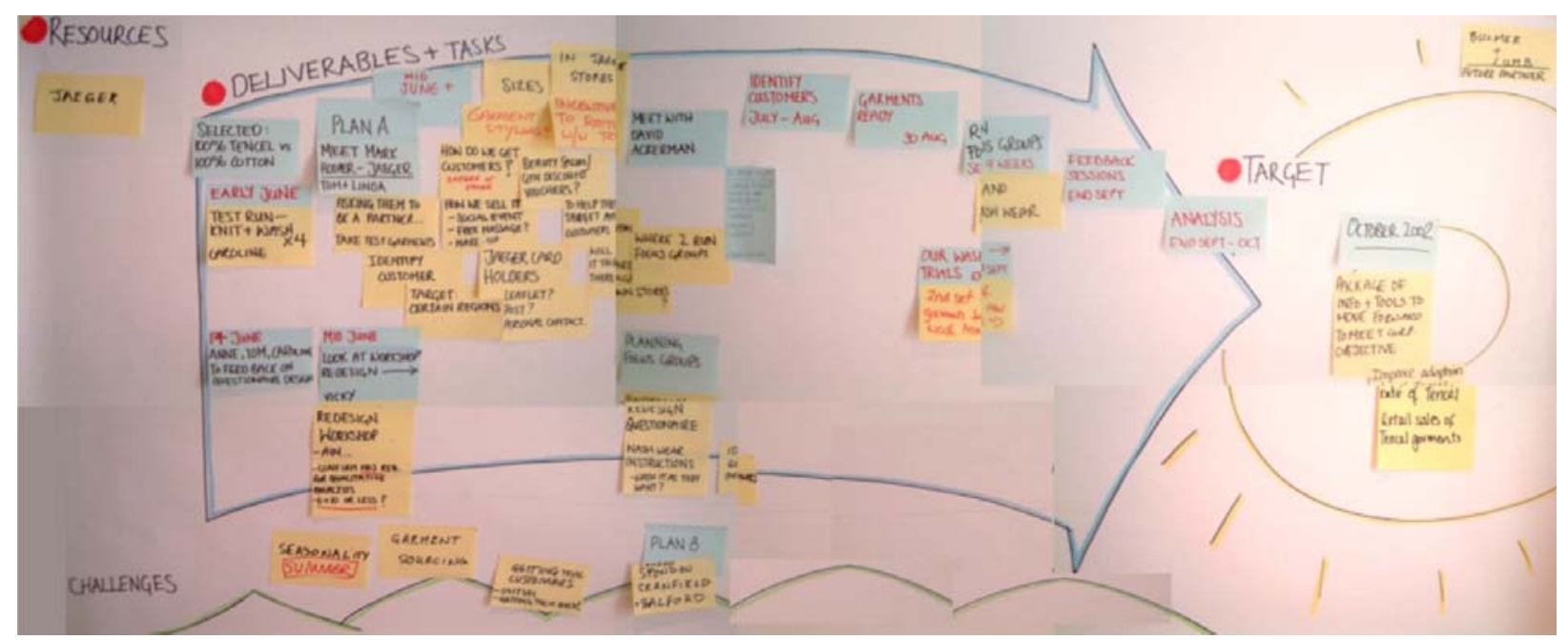

Figure 2 The Tencel 'Journey Diagram'

The 'Journey Diagram' provided many benefits which were instrumental in the successful outcome of the project. In the early stages of the project, using the template helped to draw the new team members together and provide them with a common focus. As time progressed, it helped to ensure each team member was aware of the activities that needed to be carried out, when they needed to be done and who was responsible for them. At one stage during the project a new team member joined the group and it was an easy task to brief her on the progress that had been made, the direction in which the project was headed and the reasoning behind the decisions which had been made. The template provided an effective way of making everyone accountable for their tasks in a non-confrontational and supportive way. This approach made the project very transparent and helped to ensure there were no miss-understandings within the team, which might delay its progress and threaten the tight deadline, it also enabled each team member to have a high level of project ownership, which was rewarded by commitment and enthusiasm throughout the project. In addition to this, using the template allowed the team to visually map the project over the 12-month timescale. Recording an end date from which to work back from ensured there was enough time available to complete the required activities. This was crucial to the success of the project, due to the short timescales in which we were trying to fit the later activities of the project. Finally, the visual nature of the template meant that the team could easily see the project progressing and celebrate success when the milestones were reached. It was within this context and structure that the project was carried out.

\section{$4 \quad$ Understanding the customer}

As was outlined in the introduction, collecting customer focused data was a new experience for the fibre manufacturer. In order to support this activity, the team drew from the experiences of the researchers at Cranfield University (Evans et al., 2002) who had developed empathic design tools in order to better facilitate the collection of customer focused information for Nissan. These tools, are "participatory, indepth and qualitative in nature" (Burns and Evans, 2002). Many of the tools aim to provide designers with the opportunity to "walk in the shoes of those they are designing for... to equip designers with the previously uncaptured customer information that they need to stimulate innovation, to differentiate their product and delight their customers" (Burns and Evans, 2002). In order to support this textiles project, these tools were either adapted or used as a basis for the development of new techniques, suitable for garments.

\subsection{Setting up the focus groups}

In an iterative process, the target customer for the focus groups was identified, the main aims of the process were discussed, the test garments were identified and the empathic design tools were adapted. The pilot study demonstrated that it was important to identify a specific customer base to focus on, to ensure that the tools which were to be developed were appropriate to that sector. After some initial 
investigations the Tencel/TCIF team selected a female customer group as their target audience and a profile was drawn up by the Retail Account Manager. This was used as a basis for consideration during the development of the tools (see Table 1).

\begin{tabular}{|l|l|}
\hline Female & Gardens \\
\hline Average age: $30-60$ years & Into cooking \\
\hline Middle/upper class & Self assured \\
\hline Professional/middle management & Traditional/loyal \\
\hline Family oriented & Classic look in her approach to fashion \\
\hline Travels & Environmentally aware \\
\hline
\end{tabular}

Table 1 The characteristics of the type of customer selected for the Tencel trials

In order to ensure that the activities in the focus groups generated information on the most important and appropriate areas for Tencel, the team spent some time identifying the critical areas of interest, these included;

- Customer perceptions of the Tencel garment on first sight.

- Customer perceptions of the Tencel garments in comparison with cotton.

- Customer perceptions of the comfort of the garments - short term and medium term.

- Customer perceptions of the wash performance of Tencel garments.

Through a series of discussions it was decided that the focus groups would be used to test 100\% Tencel A100 fibre variant against $100 \%$ cotton. It was felt that if the results showed that A100 was as good as cotton with similar qualities then this would provide a very strong message for Tencel. Forty test garments in $100 \%$ cotton and forty test garments in 100\% A100 were produced by a local knitwear company. Building on the findings from the pilot study, a plain black, round neck garment was developed as the test piece.

\subsection{The focus group activities and adapted tools}

Based on the interests identified above, the team developed a programme of activities that would capture customer focused information on these critical issues. Two sets of focus group activities, that would be carried out with three different sets of female' customers, over a two week period, were developed. Each focus group had an agenda of activities into which adaptations of empathic design techniques were 'woven' to achieve the desired result (see Figure 3).

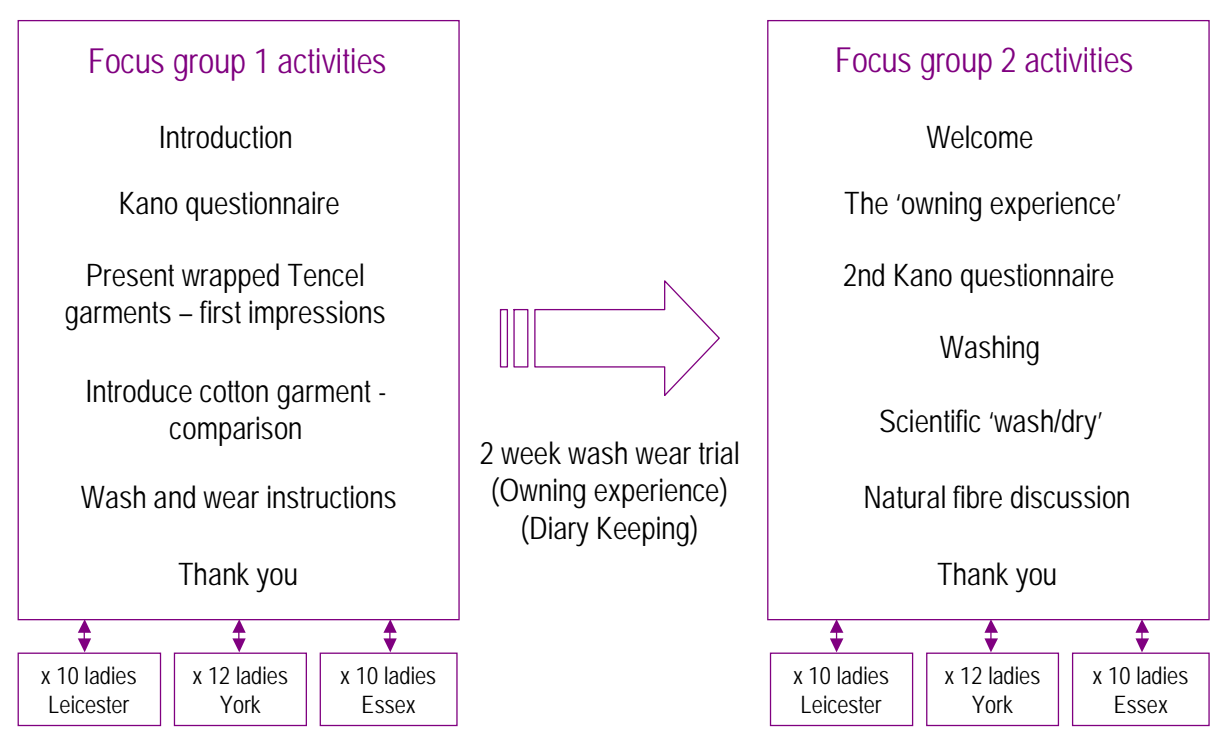

Figure 3 Outline of the Programme of activities involved in the Tencel project 
The focus groups were held in the evenings and were designed around a novel 'Cocktails and Cake' theme, with the intention of creating an atmosphere which was feminine, luxurious and fun. The meeting rooms were decorated with flowers and the participants were treated like ladies and provided with a selection of wines and beautiful cakes during the evening, to maintain a 'special' feeling. This theme evolved in response to profile data presented in Table 1. Although there were some structured activities the majority of the activities were designed to flow and encourage conversation, rather than force it. The focus groups had a relaxed and 'chatty' air and often felt more like a party than a work group.

To ensure that all opinions and feelings were captured, recording equipment was used throughout. Although the ladies were made aware of the camera and could see it through out the focus group, they often forgot about the camera and talked candidly to each other about their true feelings. This had the added benefit of providing Tencel with hard hitting evidence of exactly what the customer thought of the product - 'warts and all'. It has been seen in the past (Burns et al., 1999) and again in this project that providing a project team with this type of evidence has a very powerful effect on them, as it shows the team exactly what their customers think. This is in comparison with the diluted version which is usually provided as a result of traditional market research techniques.

\subsubsection{The Kano questionnaire}

At the beginning of the first focus group each of the particiapants were given a short questionnaire to fill in. The questionnaire which aimed to gather more information about the customer experience, was based on the Kano model (Matzler et al., 1996, Matzler and Hinterhuber, 1998) an approach which provides a way of thinking about products in terms of customer needs. To support the questionaire we asked participants to imagine that they had recently bought a knitwear garment and to consider how happy they were with the purchase, we asked them questions about various aspects of the whole life cycle of the garment in a positive way- if it had the feature, and in a negative way - if the feature was missing. The aim of this approach is to pinpoint the ladies' exact attitude to the aspect concerned. This formal activity was placed at the beginning of the evening so as not to break up the flow of activities later on.

\subsubsection{A gift wrapped present}

After the questionnaire the flow and pace of the evening changed completely and all participants were given their Tencel garment wrapped up in a beautiful package (see Figure 4).

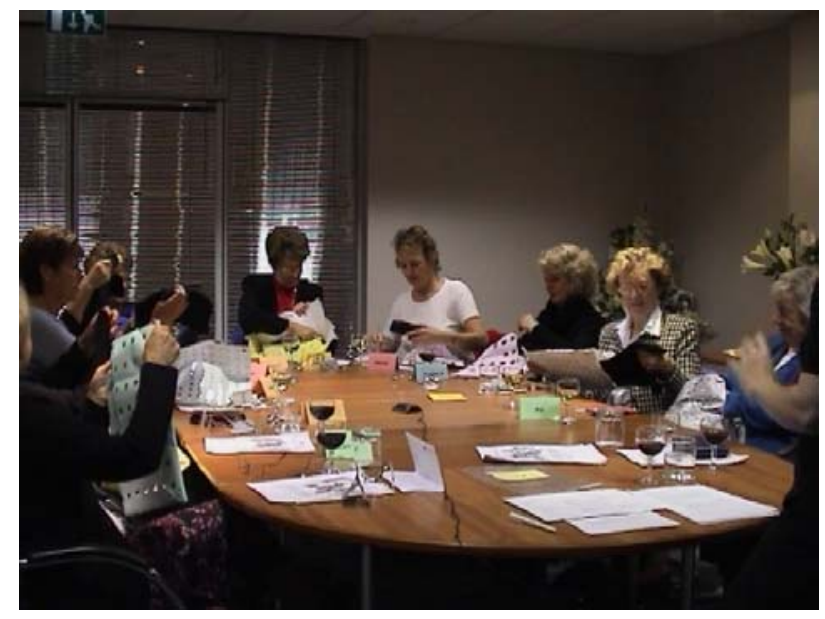

Figure 4 Opening the gift wrapped Tencel garments - 'First impressions count'

This activity was intended to provide Tencel with information on the customers 'first impressions' and was designed to replicate the experience of receiving a present. How did they feel about their gift? The initial reaction was considered to be very important to capture, as this is what sales are based on in the retail environment. The approach used at this stage drew from an empathic design technique called 'Scenario 
of Use' which aims to provide insights into how the customer thinks, feels and talks about products, by replicating specific situations that put the customer in the environment that is to be discussed.

During this activity another technique known as 'Murmur of the Customer' was used. The 'Murmur of the Customer' is a technique which captures what the customers actually think about products rather than what they tell market researchers or sales staff. In order to capture this 'murmur' at various stages during the activities, for example after the present had been unwrapped, the organisers left the room under the guise of collecting coffee, leaving the participants to chat about the garments on their own. At this stage the conversation always become more animated and candid, and the video equipment ensured that these valuable insights into their needs, wants and opinions were captured. This process ensured that comments that would not otherwise have been mentioned due to the participants 'being too polite' were captured and could subsequently be used to improve and develop the product in the future, based on more accurate data.

\subsubsection{Comparing Tencel A100 and cotton}

The next activity gave Tencel the opportunity to see how their garment compared with an identical garment knitted in cotton. The participants were given the cotton garment and asked to compare the two. It was explained that each garment was the same style and that both garments were knitted out of the same weight fibre, and dyed with the same quantity of dye. Initially during this activity the participants focused on the colour comparisons between the two. After a while they were asked to close their eyes and then talk out the difference in the garments. This was to encourage them to think about senses other than sight. Again the 'Murmur of the Customer' technique was used and the participants were left to talk in 'private'. The whole activity was recorded for analysis later on.

\subsubsection{Washing and wearing}

At the end of the first focus group the participants were introduced to the wash wear activity. The aim was that between the focus group sessions the participants would take away their new garments for two weeks and treat them as they would normally. They were asked to wash and wear them as they wanted, but to ensure that each garment was worn and washed at least once. This activity again drew on the empathic design technique of 'Scenario of Use'. It aimed to gain an insight into the customer's experiences of owning the product. The value of using this type of technique is that it enables the product developer to gain an understanding of how the actually customer feels about the product and how it actually performs, rather than making assumptions. This approach to assessing the wash/wear ability of the garment was very different to the traditional scientific approach usually taken, which involves systematically and repeatedly washing the garment using a specific washing powder and then drying it under scientific conditions.

During the two week period the participants kept a diary of their experiences, they recorded how comfortable the garments were to wear, how they wore, how they felt after washing, how they dried and any other thoughts and feelings. 'Diary Keeping' is a type of self-administered questionnaire also used to support empathic design. This approach enabled substantial amounts of data to be generated with minimal amount of effort on the part of the organisers.

At the second focus group, the same participants returned and were encouraged to talk about their experiences of owning the different products. They were encouraged to talk in groups and were subtly guided to talk about specific areas that the team had previously identified as important to capture data on. Again the activities were video recorded and the 'Murmur of the Customer' technique was used to ensure that candid opinions were captured.

During the second set of focus group the results of traditional scientific wash/wear trial, that had been run in parallel with the consumer trials, were presented to the ladies and the way in which they are tested was 
explained. This activity surprisingly raised a great deal of interest in the participants and provided a very useful insight into the issues that captured the imagination of the ladies. Again their responses were recorded on video.

To round off the evening, the participants were engaged in a conversation about their perceptions of Natural fibres and told the 'Tencel story'. This activity helped Tencel to better understand the value that their customers placed on 'natural' and provided interesting data to feed into their marketing campaigns.

\subsection{Results analysis}

Utilising and adapting the empathic design tools within a modified focus group environment enabled Tencel to collect an enormous amount of rich qualitative data from all participants. Although it was recognised that a lot of time and effort needs to go into the planning of these types of focus groups to ensure that they are a success, the resulting data means that they provide abundant return for the investment. For example as a result of this project the ladies involved provided Tencel with detailed descriptions of their first opinions of the garments, using descriptive language to express how they felt about the feel and texture of the A100 garment, such as "lovely soft feel", "lustrous", "it feels like you are putting on a cuddle". They also provided detailed opinions about the garment's drape and colour, how little it creased and the overall appearance of the garment. This level of detail and use of descriptive laungage was repeated for the cotton garment. As a result of the wash/wear trial the ladies provided detailed information on how they washed and dryed their garments and whether they referred to care labels. They also provided an insight into the performance of both garments during and after washing, in terms of texture/feel, colour performance, shrinking, stretching and creasing. Finally they provided the Tencel team with a rich perspective of the 'owning experience', providing detail on their first impressions, fit and ease of wear on both initial and subsequent wearing.

In order to identify the main findings from the project and present them to the rest of the Research and Development team at Tencel, all data arising from the questionnaires, video recordings and user diaries were transcribed and analysed using a using a coding and clustering methodology common for qualitative data (Miles and Huberman, 1994, Silverman, 2000, Yin, 1993). The main findings resulting from the analysis of the focus groups were collated into a PowerPoint presentation, and presented to an audience of representatives from the Tencel product development team which included the Head of Tencel's

Research and Development. Throughout the presentation, critical issues were illustrated with extracts of film, thus allowing the product development team to hear the true 'Voice of the Customer'. Being able to highlight the points that were made by illustrating them with video clips from the workshops, provided a very valuable way of demonstrating to the team exactly what the ladies opinions were, both positive and negative. This provide to be an extremely important exercise for the team, providing them with a real 'pat on the back' when positive comments were made and as well as highlighting where improvements were needed 'straight from the horses mouth'. The results from the project have been extremely valuable to Tencel, and are currently being fed into their Research and Development process.

\section{Conclusions}

This paper has presented the novel approach that the combined Tencel/ TCIF team took to develop a better understanding of the end customer and illustrates how techniques which were developed for one industry can be successfully adapted and applied to a quite different industry with excellent results. Using the Grove techniques helped to make the project transparent and inclusive, and enabled the whole team to be involved in the decision making process. As a result of this approach, the Tencel team have a pack of tools and activities that they were involved in developing, and the confidence to carry out future projects on their own. Drawing on the empathic design research (Evans et al., 2002) has added both interest and value to the data collection work, made it fun for the participants and enabled rich/ genuine information to be collected. Using these techniques have provided Tencel with a non-scientific way of understanding how their end customer perceives their fibre, providing unequivocal evidence of customers' true feelings 
recorded as 'raw' video based evidence. This powerful evidence means that Tencel can further strengthen their Research and Development programme based on understanding derived from the 'Voice of the Customer' rather than on preconceived, scientific ideas.

The Tencel/TCIF project has also led to a number of additional advantages for the company, such as the development of new relationships within the supply chain, the development of new relationships within Tencel and the enhancement of multi-disciplinary team working and concurrent engineering. Tencel have recognised that whilst their primary focus is to develop and manufacture fibre, there is still incredible value to be gained from talking to the end customer, and because customers think and behave very differently to scientists and engineers it is imperative to understand what is important to them from their perspective. This project has shown that the ability to identify customer delighters not only provides an excellent and powerful marketing message, but also provides sound development support for R\&D.

The powerful video evidence has provided the Tencel team with a mechanism for communication which can be used to make the senior management 'sit up and take notice' of the need to understand customers. As a consequence, a continuation project was started to work with the manufacturer who had produced the garments used with the focus groups. The target was to develop a Tencel knitwear product which could be launched on the market. Unfortunately, the manufacturer was experiencing financial difficulties and was closed by its owners shortly after the completion of the project. Hence it is not possible to point to a success that is a direct result of the project. But the methodology, the results from the focus groups and the message about the value of listening to the ultimate consumer have become engrained in the Tencel business and have been used with other customers, both retailers and knitwear manufacturers, around the world.

As a footnote, Tencel has recently been taken over by its main competitor, Lenzing. The new enlarged Lenzing business will have access to the results of this work to help develop the market for Tencel fibre.

\section{Acknowledgements}

We would like to extend our thanks to Jenny Cater, Denise Lofthouse and Chris Dowie for their help is setting up the focus groups in Essex, Leicester and York. We would also like to thank Marie Thomas at Cranfield University and our colleagues at Tencel; Linda Mears, Patricia Manning, Jim Taylor, Anne Whineray, Karen Cooper and Caroline Lavery, for their commitment to the project, and their unwavering enthusiasm to succeed.

\section{References}

Bhamra, T., Heeley, J. and Tyler, D. (1998) A Cross Sectoral approach to New Product Development. The Design Journal, 1(3): pp. 2-16.

Burns, A., Barrett, R., Evans, S. and Johansson, C. (1999) Delighting Customers Through Empathic Design. In 6th International Product Development Management Conference, Cambridge, UK, 56th July 1999.

Burns, A. and Evans, S. (2000) "Insights into Customer Delight." (Eds, Scrivener, S. A. R., Ball, L. J. and Woodcock, A.) Chapter 29 in Collaborative Design. London: Springer.

Burns, A. and Evans, S. (2002) Empathic Design: A New Approach for Understanding \& Delighting Customers. International Journal of New Product Development and Innovation Management, 3(4).

Burns, A., Evans, S., Johansson, C. and Barrett, R. (2000) An Investigation of Customer Delight During Product Evaluation. In 7th International Product Development Management Conference, Leuven, Belgium, 29-30th May 2000.

Curtis, H. (1997) Implementation of Environmentally Conscious Design in Courtaulds Fibres: Tencel. Manchester Metropolitan University, Manchester, 160.

Evans, S., Burns, A. and Barrett, R. (2002) Empathic Design Tutor. Cranfield: Cranfield University. ISBN 1 861940866 
Fashion Industry Forum (2002) "Website of the Fashion Industry Forum". www.industryforum.net (September 2002).

Lofthouse, V. A. and Thomas, M. (2002) Understanding customer perceptions in knitwear, Cranfield University, Cranfield, pp.1-61. Internal report for Tencel Ltd. (unpublished)

Matzler, K. and Hinterhuber, H. H. (1998) How to make product development projects more successful by integrating Kano's model of customer satisfaction into quality function deployment. Technovation, 18(1): pp. 25-38.

Matzler, K., Hinterhuber, H. H., Bailom, F. and Sauerwein (1996) How to delight your customers. Journal of Product \& Brand Management, 5(2): pp. 6-18.

Miles, M. B. and Huberman, A. M. (1994) An Expanded Sourcebook - Qualitative Data Analysis.: Sage Publications.

Silverman, D. (2000) Doing Qualitative Research: A Practical Handbook. London: Sage Publications.

Tencel Ltd. (2002) "Experience More. Experience TENCEL®". http://www.tencel.com/ (25th June 2003) Tencel Ltd.,. Company website

The Grove Consultants International (2001) "A Graphic Facilitation Retrospective: Charting What We Learned". www.grove.com/new/new_gfretro.html (June 2001).

The Grove Consultants International (2003) "The Grove Consultants International". www.grove.com (18th June 2003).

Yin, R., K. (1993) Applications of Case Study Research. Vol. Volume 34 London: Sage.

Young, K., Lettuce, F. and Leslie, A. (2003) Methods for Facilitation and Collaboration, Cranfield University. 